\title{
The Concept Study of Recombinant Human Soluble Thrombomodulin in Patients with Acute Respiratory Distress Syndrome
}

\author{
Kenji Tsushima $^{1,3^{*}}$, Toshiki Yokoyama², Tomonobu Koizumi ${ }^{2}$, Keishi Kubo ${ }^{2}$, Koichiro Tatsumi ${ }^{3}$ \\ ${ }^{1}$ Department of Pulmonary Medicine, Shinonoi General Hospital, Nagano, Japan; ${ }^{2}$ First Department of Internal Medicine, Shinshu \\ University School of Medicine, Nagano, Japan; ${ }^{3}$ Department of Respirology, Graduate School of Medicine, Chiba University, Chiba \\ Japan. \\ Email: *tsushimakenji@yahoo.co.jp
}

Received August $27^{\text {th }}, 2013$; revised September $28^{\text {th }}, 2013$; accepted October $15^{\text {th }}, 2013$

Copyright (C) 2013 Kenji Tsushima et al. This is an open access article distributed under the Creative Commons Attribution License, which permits unrestricted use, distribution, and reproduction in any medium, provided the original work is properly cited.

\begin{abstract}
Background: Recombinant human soluble thrombomodulin (rhTM) was approved for the treatment of disseminated intravascular coagulation in Japan, and rhTM has anti-inflammatory effects. Disordered coagulation is a part of the acute respiratory distress syndrome (ARDS) pathophysiology and thus we hypothesize that anticoagulant therapy may help. This preliminary study was to observe the safety of rhTM administration and the improvement on biomarker levels after the therapy for ARDS-patients. Objectives: Case series of ARDS-patients. Methods: Seventeen ARDS-patients that required ventilatory management were treated with rhTM and clinical and laboratory data were collected including platelets, thrombin-antithrombin complex (TAT), fibrinogen degradation products, oxygen saturation/the fraction of inspired oxygen $\left(\mathrm{SpO}_{2} / \mathrm{F}_{1} \mathrm{O}_{2}\right)$, and high-mobility group-1 (HMG-1). The administration of rhTM was started during 6 days at a bolus dose of $0.06 \mathrm{mg} / \mathrm{kg} /$ day immediately after the diagnosis of ARDS. Results: Eleven of the 17 ARDS-patients were alive at 28 days after the beginning of the administration of rhTM. The serial pattern of the $\mathrm{SpO}_{2} / \mathrm{F}_{\mathrm{I}} \mathrm{O}_{2}$ showed remarkable differences between the survivors and nonsurvivors from day 5 to day 7 . The TAT in the survivors significantly decreased after treatment, and there were significantly lower levels in the TAT on day 7 in comparison to that of the nonsurvivors. The serial changes of HMG-1 showed increased levels in the nonsurvivors until day 5 after the administration of rhTM. Conclusions: Additional rhTM administration can safely improve the parameters in survival ARDS-patients, as demonstrated by significant improvements in the $\mathrm{SpO}_{2} / \mathrm{F}_{1} \mathrm{O}_{2}, \mathrm{HMG}-1$ and TAT.
\end{abstract}

Keywords: Acute Respiratory Distress Syndrome; Recombinant Human Soluble Thrombomodulin; Thrombin-Antithrombin Complex; $\mathrm{SpO}_{2} / \mathrm{F}_{\mathrm{I}} \mathrm{O}_{2}$; High-Mobility Group-1

\section{Introduction}

Thrombomodulin (TM) is a transmembrane protein expressed on the endothelial cell surface that plays an important role in the regulation of intravascular coagulation [1]. The novel biological agent, recombinant human soluble thrombomodulin (rhTM), was approved and is being used clinically for the treatment of disseminated intravascular coagulation (DIC) in Japan. The effects of rhTM on DIC were previously examined in a multicenter, randomized clinical trial in Japan [2], and the resolution of DIC was significantly better in the group treated with rhTM than in the group treated with unfractionated heap-

${ }^{*}$ Corresponding author. rin. The rhTM binds to thrombin to inactivate coagulation, and the thrombin-rhTM complex activates protein $\mathrm{C}$ to produce activated protein $\mathrm{C}$ (APC), which, in the presence of protein $\mathrm{S}$, inactivates factors VIIIa and Va, thereby inhibiting the further thrombin formation.

Acute respiratory distress syndrome (ARDS) is also characterized by excessive intra-alveolar fibrin deposition, driven at least partly by inflammation [3]. The imbalance between the activation of coagulation and inhibition of fibrinolysis in patients with ARDS appears to occur both systemically in the lungs and the alveoli [4]. The activation of tissue factor (TF) is a critical event that results in thrombin formation. Thrombin is a key intermediate molecule with several biological functions, in- 
cluding the augmentation of vascular permeability [5] and enhancement of inflammation [6]. Thrombin generation leads to fibrin polymerization and deposition, with the resultant formation of hyaline membranes and a pathological hallmark of ARDS. The modulation of coagulation and fibrinolysis has a complex effect on both hemostatic and inflammatory pathways. Therefore, anticoagulation therapy is beginning to be recognized as a potential new strategy for the treatment of ARDS patients.

The aim of the present preliminary study was to evaluate whether the additional administration of rhTM has the improvement in biomarker levels with the onset of therapy between survival and nonsurivival patients with ARDS.

\section{Methods}

Our study was approved by the ethics committee of Shinonoi General Hospital, and all patients or their family gave written informed consent on admission. We performed a prospective study of adult patients with ARDS who were admitted between April 2010 and September 2012. Our clinical study was registered to Shinonoi General Hospital, Chikuma Central Hospital and Shinshu University School of Medicine.

\section{The Diagnosis of ARDS}

The criteria for the diagnosis of ARDS, as set by the Berlin Definition [7], were used as follows: acute onset of lung injury, diffuse bilateral infiltrates seen upon chest $\mathrm{X}$-ray, a $\mathrm{PaO}_{2} / \mathrm{F}_{\mathrm{I}} \mathrm{O}_{2}(\mathrm{PF})$ ratio for ARDS, pulmonary artery occlusion pressure $<19 \mathrm{mmHg}$, or no clinical evidence of congestive heart failure. To rule out congestive heart failure, we performed echocardiographic examinations. Enrolled patients were diagnosed as having ARDS on admission.

\section{Noninvasive Ventilation and Endotracheal Intubation}

Enrolled patients were performed chest X-ray on admission. All patients initially received oxygen via a nasal cannula to maintain an oxygen concentration of more than $60 \mathrm{mmHg}$. If they could not maintain oxygen saturation $\left(\mathrm{SpO}_{2}\right)$ of more than $90 \%$ or an oxygen concentration $\left(\mathrm{PaO}_{2}\right)$ of more than $60 \mathrm{mmHg}$ with $3-5 \mathrm{~L} /$ minute of oxygen via the nasal cannula, we recommended noninvasive ventilation (NIV) with an oronasal mask and bi-level positive airway pressure ventilation (BiPAP) with a BiPAP Vision device (Respironics; Murrysville, PA). All patients received NIV with the bilevel mode at a maximal positive pressure of $30 \mathrm{cmH}_{2} \mathrm{O}$. The spontaneous timing mode was used for all patients, with a back-up rate of 10 breaths/minute. Inspiratory positive airway pressure (IPAP) was commenced at $4 \mathrm{~cm} \mathrm{H}_{2} \mathrm{O}$, and adjusted to achieve a respiratory rate of less than 30 breaths/minute. The expiratory positive airway pressure (EPAP) was adjusted to achieve the target level of oxygenation with minimum carbon dioxide rebreathing. The oronasal mask or full face mask was fitted by a trained pulmonologist. Straps were adjusted to allow one finger to pass easily between the strap and the patient's face. The fraction of inspired oxygen $\left(\mathrm{F}_{\mathrm{I}} \mathrm{O}_{2}\right)$ and IPAP were adjusted to maintain a $\mathrm{SpO}_{2}$ of more than $90 \%$ or a $\mathrm{PaO}_{2}$ of more than $60 \mathrm{mmHg}$.

Initial settings for NIV were chosen empirically by the attending pulmonologists. Patients were initiated on IPAP of $4 \mathrm{cmH}_{2} \mathrm{O}$ and a $\mathrm{F}_{\mathrm{I}} \mathrm{O}_{2}$ of $100 \%$, after which the NIV was titrated by the pulmonologist to maintain patient comfort and to keep the respiration rate at $<35$ breaths/minute, $\mathrm{pH}>7.3$, and oxygen saturation $>90 \%$.

If the patients could not maintain an oxygen saturation of more than $90 \%$ or an arterial oxygen concentration of more than $60 \mathrm{mmHg}$ with the BiPAP Vision at 2 hours and 24 hours after initiation of NIV, we recommended endotracheal intubation. Endotracheal intubation was performed for patients with decreased alertness or major agitation requiring sedation, clinical signs of exhaustion (active contraction of the accessory muscles with paradoxical abdominal or thoracic motion), hemodynamic instability, cardiac arrest, refractory hypoxemia, or a $\mathrm{PaO}_{2} / \mathrm{F}_{\mathrm{I}} \mathrm{O}_{2}$ (PF) ratio of less than 100 after treatment with NIV. When an improvement of the respiration rate and a decrease of the oxygen requirement became evident, the patients were gradually weaned off ventilation during the daytime and then during sleeping hours. Patient or family refusal of invasive mechanical ventilation was an exclusionary criterion for endotracheal intubation. These patients were excluded from this study.

\section{Treatment}

Standard microbiological investigations (e.g., blood and sputum cultures) were performed before the start of treatment to exclude pulmonary infection. Bronchoalveolar lavage (BAL) was performed at admission, except in patients with refractory hypoxaemia despite sufficient mechanical ventilation, those with hemodynamic instability, and those who rejected BAL. BAL fluid was assessed to exclude infectious disease. The administration of rhTM (recomodulin ${ }^{\circledR}$ ) was started during 6 days at a bolus dose of $0.06 \mathrm{mg} / \mathrm{kg} / \mathrm{day}$. Microbiological investigations (e.g., blood and sputum cultures) were performed before the start of antibiotic therapy. The exclusion of this study was done on suspicion of infection at admission when the microbiological cultures were assessed as positive. Broad-spectrum antibiotics were then administered until the offending pathogen was identified for all 
ARDS patients.

\section{Data Collection}

rhTM administration can use for 6 days, and is effective for acute phase of ARDS patients. Therefore, patients were followed until 28 days after entry into the study. Patients were defined as survivors and nonsurvivors if they were alive or dead 28 days after beginning rhTM treatment, respectively. The collected data were age, sex, PF ratio, $\mathrm{SpO}_{2} / \mathrm{F}_{1} \mathrm{O}_{2}$, Sequential Organ Failure Assessment (SOFA) score and DIC score on admission. We evaluated the 28-day mortality after admission, and the physiological and biochemical variables. White blood cell counts (WBC, normal range: $4000-9000 / \mu \mathrm{L}$ ), platelet counts (normal range: $14-30 \times 10^{4} / \mu \mathrm{L}$ ), the serum lactate dehydrogenase level (LDH, normal range: 120 - 235 $\mathrm{IU} / \mathrm{L}$ ), fibrinogen degradation products (FDP, normal range: less than $5 \mu \mathrm{g} / \mathrm{mL}$ ), and C-reactive protein (CRP, normal range: less than $0.3 \mathrm{mg} / \mathrm{dl}$ ) were recorded on days 0 (before), 1, 2, 3, 5, and 7 after admission. The Krebs von den Lungen-6 (KL-6, normal range: less than 500 $\mathrm{U} / \mathrm{mL}$ ) and thrombin-antithrombin complex (TAT, normal range: less than $4 \mathrm{ng} / \mathrm{ml}$ ) values were recorded on days 0 (before), and 7 after admission. Cytokines (interleukin-6 (IL-6, normal range: less than $4.0 \mathrm{pg} / \mathrm{mL}$ ), and high-mobility group-1 (HMG-1) protein were measured on days 0 (before), 1,3 , and 7 after starting the treatment. The presence of serious adverse events was defined as follows: fatal bleeding, nonfatal serious bleeding (defined as intracranial hemorrhage confirmed by brain imaging, gastrointestinal or respiratory tract bleeding uncontrollable by conservative treatments, and bleeding at a critical location, such as retinal hemorrhage, major hemarthrosis or spinal hemorrhage) or any life-threatening bleeding that led to discontinuation of the administered study drug. The $\mathrm{SpO}_{2} / \mathrm{F}_{1} \mathrm{O}_{2}$ ratio was calculated at 1 hour and on days $1,2,3,5$, and 7 after initiation of NIV on behalf of the PF ratio.

\section{Outcomes}

The primary outcome was whether rhTM administration was safe treatment without adverse events for ARDS patients. The secondary outcome is whether the additional administration of rhTM has a beneficial effect in biomarker levels with the onset of therapy for patients with ARDS.

\section{Statistical Analysis}

The data are expressed as the group means plus or minus the standard error. Continuous variables were compared between groups by using Student's $t$-tests. Categorical variables were analyzed by using Fisher's exact test. A univariate analysis of the time to mortality was compared by using a log-rank test. The comparisons of the WBC and platelet counts, CRP, FDP, $\mathrm{LDH}, \mathrm{SpO}_{2} / \mathrm{F}_{\mathrm{I}} \mathrm{O}_{2}$, IL-6, and HMG-1 between groups over time were analyzed by repeated measure analysis of variance (ANOVA) adjusted for the baseline values as a covariate. A $p$ value $<$ 0.05 was considered to be statistically significant.

\section{Results}

\subsection{Baseline Characteristics}

Seventeen patients with ARDS were enrolled. The etiology of ARDS patients was 12 direct ARDS including 3 aspiration pneumonias and 5 indirect ARDS patients with sepsis. The bacteria or fungus in the BAL were not detected. Their mean age was $75.5 \pm 19.9$ years. Enrolled ARDS patients were included in 1 mild ARDS, 12 moderate ARDS and 4 severe ARDS patients. Table 1 showed that thrombosis showing elevated plasma TAT and FDP levels in the pulmonary vasculature due to lung inflammation was present in patients with ARDS.

\subsection{The Effects of Treatment on Mortality}

The 28 -day mortality rate was $35.3 \%$. The cause of death among the patients who died was the deterioration of ARDS. Enrolled patients were classified into survivors and nonsurvivors at 28 day after administration of rhTM. Table 1 showed that endotracheal intubation was required for 13 with ARDS, and NIV was performed for 4 patients with ARDS. Nonsurvivors showed significantly increased levels of TAT on admission compared to that of survivors $(\mathrm{P}=0.0081)$. Five of the 6 nonsurvivors showed high levels of serum KL-6 on admission, without fibrosis and traction bronchiectasis on chest HRCT. ARDS patients were classified into high KL-6 (abnormal levels, more than $500 \mathrm{U} / \mathrm{mL}$ ) and normal KL-6 subgroups according to their serum KL-6 levels on admission. There was a significant difference in the mortality rate between normal KL-6 and high KL-6 groups $(\mathrm{P}=$ 0.005) (Figure 1).

\subsection{The Effects of Treatment on the Clinical Data in the Patients with ARDS}

In the serial changes in survivors, there was a significant difference in the WBC counts (Figure 2(a)) on days 5 and 7 as compared to that in nonsurvivors $(\mathrm{P}=0.024$ and 0.0076 , respectively). There were no significant differences in the serial changes in the CRP (Figure 2(b)) and LDH (Figure 2(c)) from before treatment until day 7 between the two groups. The $\mathrm{SpO}_{2} / \mathrm{F}_{\mathrm{I}} \mathrm{O}_{2}$ (Figure 2(d)) rapidly increased on 1 hour in survivors, and increased from 
Table 1. Characteristics on admission.

\begin{tabular}{|c|c|c|c|}
\hline & Survivor $(n=11)$ & Nonsurvivor $(\mathrm{n}=6)$ & $P$ value \\
\hline Age (years) & $73.7 \pm 4.3$ & $77.2 \pm 1.2$ & 0.66 \\
\hline Sex, M/F & $8 / 3$ & $4 / 2$ & 0.40 \\
\hline Mild ARDS & 1 & 0 & \\
\hline Moderate ARDS & 8 & 4 & \\
\hline Severe ARDS & 2 & 2 & \\
\hline Direct/Indirect & $7 / 4$ & $5 / 1$ & 0.39 \\
\hline Infectious/Noninfectious & $8 / 3$ & $6 / 0$ & 0.16 \\
\hline $\mathrm{WBC}(/ \mu \mathrm{L})$ & $15216 \pm 1232$ & $15640 \pm 1181$ & 0.77 \\
\hline Platelet $\left(\times 10^{4} / \mu \mathrm{L}\right)$ & $15.2 \pm 2.4$ & $20.1 \pm 3.9$ & 0.52 \\
\hline Fibrinogen (mg/dL) & $397 \pm 45.7$ & $501 \pm 76.8$ & 0.57 \\
\hline $\mathrm{PT}(\mathrm{sec})$ & $1.15 \pm 0.02$ & $1.68 \pm 0.10$ & 0.22 \\
\hline APTT (sec) & $29.9 \pm 0.29$ & $41.5 \pm 1.2$ & 0.038 \\
\hline FDP $(\mu \mathrm{g} / \mathrm{mL})$ & $45.2 \pm 4.4$ & $164 \pm 39.1$ & 0.16 \\
\hline TAT (ng/mL) & $22.7 \pm 2.1$ & $49.6 \pm 2.6$ & 0.0081 \\
\hline LDH (IU/L) & $468 \pm 45.1$ & $866 \pm 109$ & 0.16 \\
\hline CRP (mg/mL) & $13.2 \pm 2.3$ & $17.7 \pm 2.0$ & 0.46 \\
\hline KL-6 (U/mL) & $403 \pm 62.4$ & $656 \pm 46.8$ & 0.26 \\
\hline SOFA score & $8.1 \pm 0.3$ & $7.8 \pm 0.6$ & 0.81 \\
\hline DIC score & $5.5 \pm 0.3$ & $5.2 \pm 0.6$ & 0.82 \\
\hline $\mathrm{PF}$ ratio $(\mathrm{mmHg})$ & $145 \pm 8.5$ & $107 \pm 5.3$ & 0.14 \\
\hline $\mathrm{SpO}_{2} / \mathrm{F}_{\mathrm{I}} \mathrm{O}_{2}(\%)$ & $155 \pm 9.3$ & $142 \pm 6.6$ & 0.44 \\
\hline Intubation/NIV (n) & $9 / 2$ & $4 / 2$ & 0.40 \\
\hline
\end{tabular}

AE-IPF, acute exacerbation of idiopathic pulmonary fibrosis; ARDS, acute respiratory distress syndrome; M, male; F, female; WBC, w8hite blood cell; PT, prothrombin time; APTT, activated partial thromboplastin time; FDP, fibrinogen degradation products; TAT, thrombin-antithrombin complex; LDH, lactate dehydrogenase; CRP, C-reactive protein; KL-6, Krebs von den Lungen-6; SOFA, Sequential Organ Failure Assessment; DIC, disseminated intravascular coagulation; $\mathrm{PF}, \mathrm{PaO}_{2} / \mathrm{F}_{\mathrm{I}} \mathrm{O}_{2} ; \mathrm{NIV}$, noninvasive ventilation.

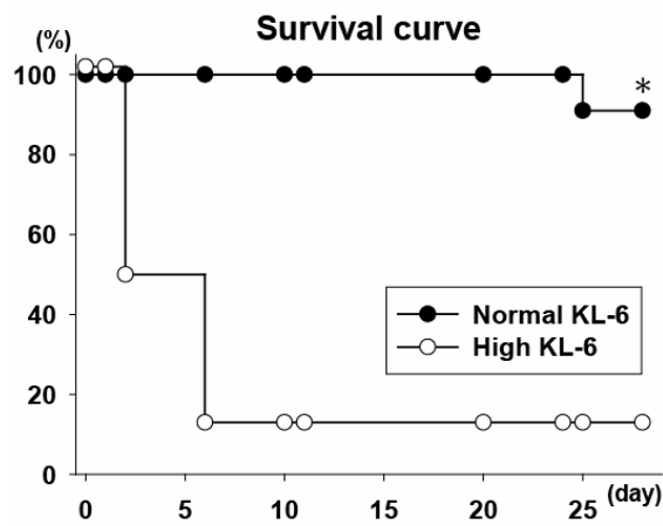

Figure 1. A comparison of the survival curves. The survival curves of normal KL-6 ARDS and high KL-6 ARDS patients. The ARDS patients were classified into high KL-6 and normal KL-6 groups on the basis of their initial serum KL-6 levels. There was a significant difference in the mortality rate between normal KL-6 and high KL-6 patients ( $P$ $=0.045) .{ }^{*} P<0.05$ compared to the high KL-6 ARDS group. KL-6, Krebs von den Lungen-6.

day 3 in survivors, and continued to improve until day 7 . There was a significantly higher $\mathrm{SpO}_{2} / \mathrm{F}_{\mathrm{I}} \mathrm{O}_{2}$ in survivor compared to that of nonsurvivors on day 5 and 7 after treatment $(\mathrm{P}=0.049$ and 0.046 , respectively).

\subsection{The Effect of Treatment on the Coagulation Data in the Patients with ARDS}

The serial changes of coagulation data in the patients with ARDS are shown in Figure 3. There were no significant differences in the serial changes in the platelet counts (Figure 3(a)) and fibrinogen (Figure 3(b)) between the two groups. The serial changes of the FDP in nonsurvivors were tended to be higher than those of survivors (Figure 3(c)). There was a significant difference in the serial change of the fibrinogen and TAT in survivors (Figures 3(b) and 3(d)) ( $\mathrm{P}=0.015$ and 0.039 , respectively) on day 7 compared to the initial data. In survivors, TAT showed significantly lower levels in comparison to that of nonsurvivors on admission and on day 7 after treatment of $\operatorname{rhTM}(\mathrm{P}=0.0081$ and 0.0089 , respectively).

\subsection{Cytokine Levels after the Administration of rhTM}

The serum IL-6 (Figure 4(a)) decreased in survivors, and 

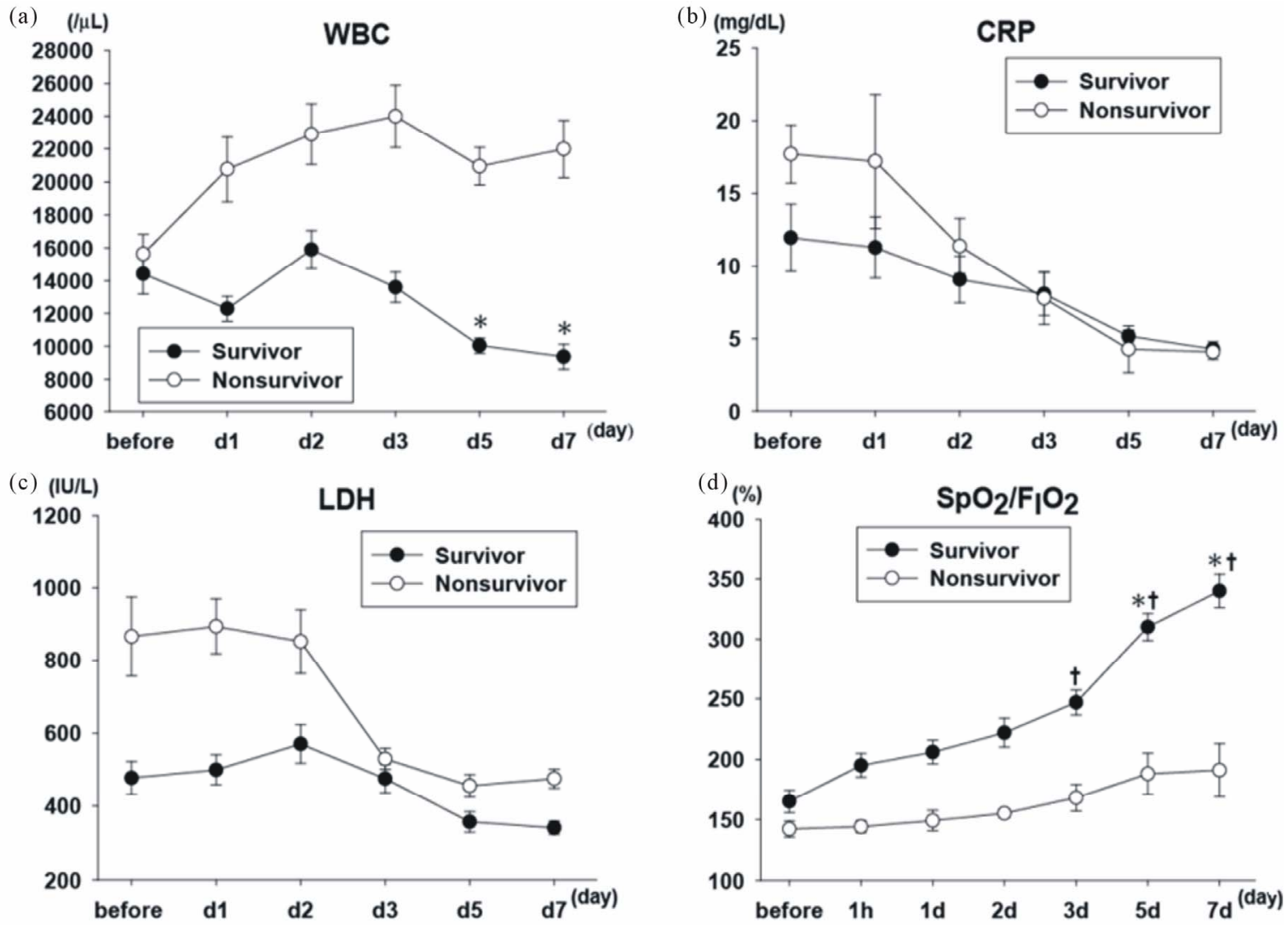

Figure 2. The serial changes of the WBC count, CRP, LDH and $\mathrm{SpO}_{2} / \mathrm{F}_{\mathrm{I}} \mathrm{O}_{2}$. The data are expressed as the group means \pm standard error of the mean. (a) The serial changes of the WBC count in survivor were significant difference on day 5 and 7 in comparison to nonsurvivor. (b) There were not significant differences in the change of the CRP level between two groups. (c) There were no significant differences in the changes in the LDH level between the two groups. (d) $\mathrm{The} \mathrm{SpO}_{2} / \mathrm{F}_{\mathrm{I}} \mathrm{O}_{2}$ increased from day 3 in survivor, and continued to improve until day 7 . There was a significantly higher $\mathrm{SpO}_{2} / \mathrm{F}_{\mathrm{I}} \mathrm{O}_{2}$ in survivor compared to that of nonsurvivor on day 5 and 7 after treatment. ${ }^{*} \mathbf{P}<0.05$ compared to the nonsurvivor, ${ }^{\dagger} \mathbf{P}<0.05$ compared to before treatment. WBC, white blood cell; CRP, c-reactive protein; $\mathrm{LDH}$, lactate dehydrogenase; $\mathrm{SpO}_{2} / \mathrm{F}_{\mathrm{I}} \mathrm{O}_{2}$, oxygen saturation/the fraction of inspired oxygen.

was closer to the initial levels in nonsurvivors. The IL-6 level in survivor on day 7 was significantly decreased compared to the initial level $(\mathrm{P}=0.019)$. The serial changes of HMG-1 (Figure 4(b)) showed increased levels in nonsurvivors until day 7 after administration of rhTM. On day 7, the HMG-1 level in nonsurvivors was significantly higher compared to the initial level in nonsurvivosr and the level on day 7 in survivors $(\mathrm{P}=0.011$ and 0.0002 , respectively). Serum KL-6 on day 7 in survivor was a significantly lower level than that of nonsurvivor $(P=0.027)$, and serum KL-6 on day 7 in nonsurvivors was a significant higher levels than that on admis$\operatorname{sion}(\mathrm{P}=0.049)$.

\subsection{Adverse Events}

No adverse events according to bleeding had after the administration of rhTM. There were no other potential adverse events, such as liver, renal function, or cardio- vascular systems.

\section{Discussion}

Our present study suggests that activated intravascular coagulation disturbances occurred in ARDS patients because of elevated levels of plasma FDP and TAT. The modulation of the coagulation disturbance by our conventional therapy with rhTM administration may be related to the mortality of ARDS patients, because the serial changes in the fibrinogen and plasma TAT, and the $\mathrm{SpO}_{2} / \mathrm{F}_{\mathrm{I}} \mathrm{O}_{2}$ level showing the respiratory conditions significantly increased in ARDS patients. We found additional rhTM administration appeared to be safe, without any major adverse effects.

TF expression, a key mediator of the activation of coagulation in the lungs, and fibrin deposition, were detected by the presence of specific antibodies in the alveolar space and the pathogenesis of the early phase of 

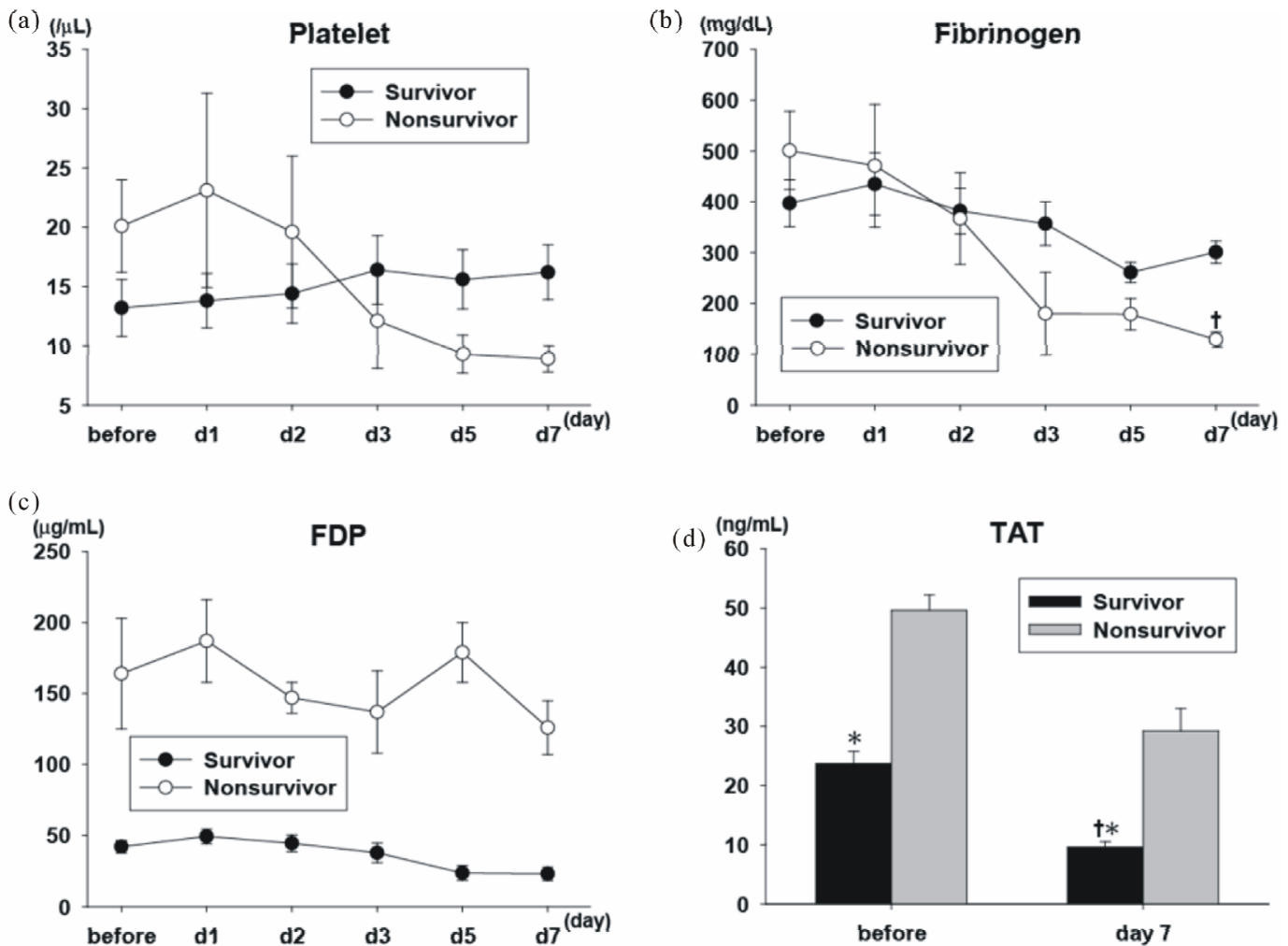

Figure 3. The serial changes in the coagulation data. The data are expressed as the group means \pm standard error of the mean. (a) There were no differences in the serial changes in the platelet counts between the two groups. (b) There was a significantly lower level in nonsurvivor compared to that on day 7 after treatment. (c) There were no differences in the serial changes in the plasma FDP between the two groups. (d) The plasma TAT showed significantly lower levels in survivors before the administration of rhTM. On day 7, the plasma TAT level in survivors was significantly lower that the initial level and the level of nonsurvivors ${ }^{*} \mathbf{P}<\mathbf{0 . 0 5}$ compared to the nonsurvivor, ${ }^{\dagger} \mathbf{P}<\mathbf{0 . 0 5}$ compared to before treatment. FDP, fibrinogen degradation products; TAT, thrombin-antithrombin complex.

ARDS [3]. The imbalance between the activation of coagulation and inhibition of fibrinolysis in patients with ARDS appears to occur both systemically and in the alveolar space. Therefore, rhTM, which can modulate the coagulation pathway, such as the protein $\mathrm{C}$ pathway and the antithrombin pathway, may be useful for the treatment of ARDS.

The use of activated protein $\mathrm{C}$ for ARDS patients, however, was a negative trial [8]. The rhTM plays an important role in regulating not only coagulation, but also inflammation, under conditions of sepsis. Two different mechanisms have been described for the anticoagulant effects of rhTM [1,9]. Thrombin is one of the key molecules involved in coagulation, and has several biological functions, including augmentation of vascular permeability [5] and enhancement of inflammation [6]. Thrombin formation leads to fibrin polymerization and deposition, with resultant formation of hyaline membranes, a pathological hallmark of ARDS. Direct binding of rhTM to thrombin can lead to its disruption. Therefore, the major mechanism of rhTM binding to thrombin is a pathway through the production of activated protein $\mathrm{C}$.
Recently, another mechanism underlying the anti-inflammatory effect has been reported, wherein the N-terminal lectin-like domain of rhTM sequesters and cleaves HMG-1, which is released from necrotic cells and modulates several signals that induce a proinflammatory response leading to severe cell damage [10-12] and by lipopolysaccharide in the plasma in an experimental endotoxin model [11]. Nagato et al. reported that rhTM inhibits the production of inflammatory cytokines, decreases the plasma HMG-1 levels and reduces the mortality in experimental endotoxemia in rats [13]. In the present study, the levels of serum IL-6, as inflammatory cytokines, were significantly increased in non-survivors on day 7 after administration of rhTM, and the HMG-1 in non-survivors could not be suppressed by increasing the administration of rhTM. Supporting their poorer prognosis, Abraham et al. described that an increase in HMG-1 may be related to the development of lung inflammation, resulting in mortality [14]. Moreover, the mortality of ARDS patients was related to the development of fibrosis related to the increased levels of serum KL-6 [15]. In our study, serum KL-6 level was related to the mortality and 

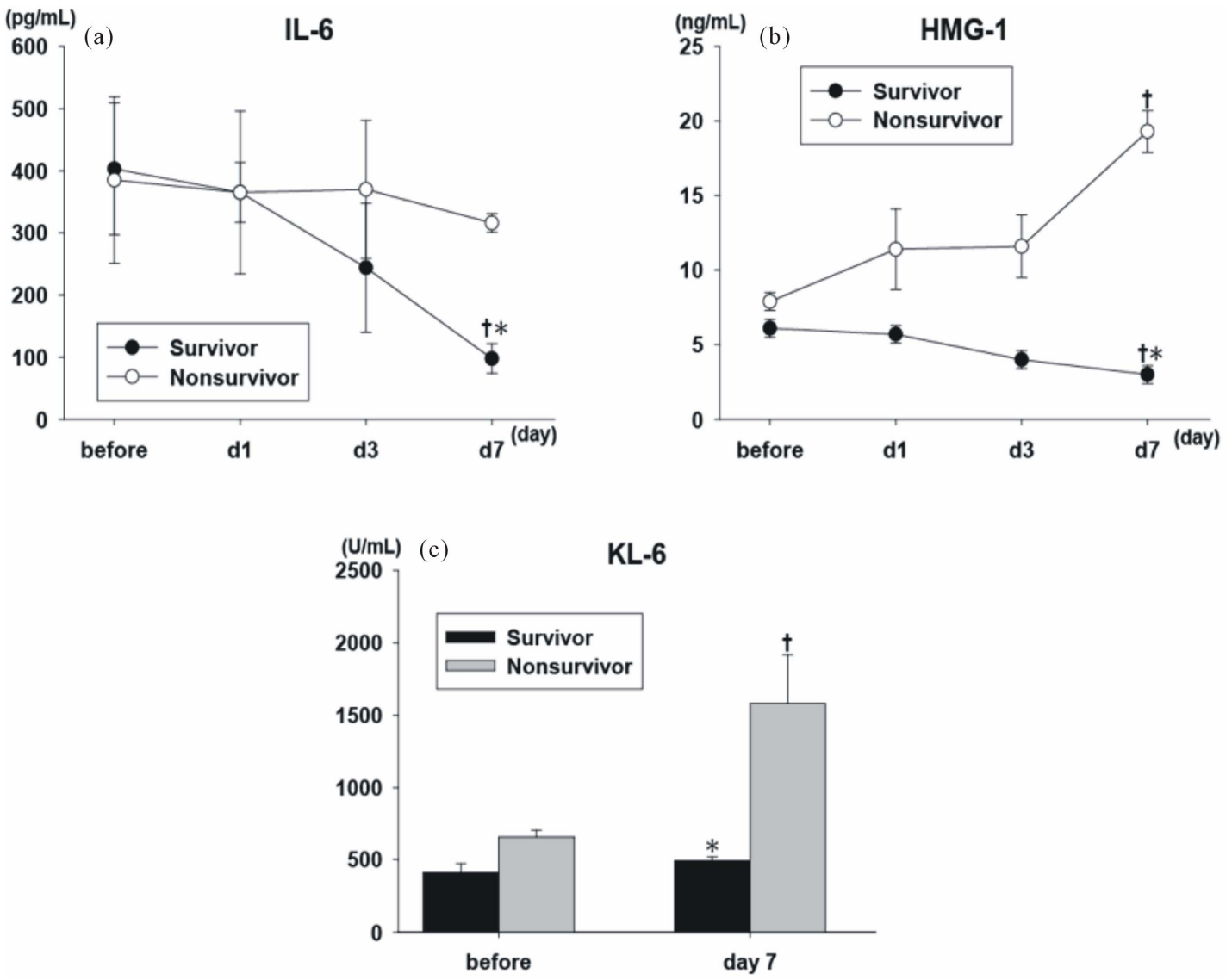

Figure 4. The serial changes in the inflammatory cytokines and serum KL-6 in survivors and nonsurvivors. The data are expressed as the group means \pm standard error of the mean. The serum IL-6 (a) decreased until day 7 in survivor, and was close to the initial levels in nonsurvivor compared to survivors. (b) The serial changes of HMG-1 showed increased levels in nonsurvivor until day 7 after the administration of rhTM. On day 7, the HMG-1 level in nonsurvivor was significantly higher that the initial level and the level of survivors. (c) on day 7, serum KL-6 levels in survivor showed significant lower compared to that of nonsurvivor and serum KL-6 levels in nonsurvivor showed significant higher compared to before the administration of rhTM. ${ }^{*} \mathbf{P}<0.05$ compared to the nonsurvivor, ${ }^{\dagger} \mathbf{P}<0.05$ compared to before treatment. IL-6, interleukin-6; HMG-1, high-mobility group-1 protein; KL-6, Krebs von den Lungen-6.

therapeutic effects (Figures 1 and 4(c)). The six patients who died in our study showed worse results in most of the biomarker levels although they received the same treatment as the survival ARDS patients. We interpret them as a phenomenon of non-responders. As shown in Figure 1, the oxygenation has been shown to be a significant predictor of responders because of the deterioration of their lung parenchyma [16].

There were a few limitations to our preliminary study. First, we could not obtain lung tissue specimens to confirm the existence of microvascular thrombus formation in the lungs of ARDS patients before and after treatment. We speculated that intra-alveolar thrombin formation and fibrin deposition in the local alveoli occurs at the same time as when the systemic thrombin formation develops in blood. Second, the study involved a small number of patients, and it was also a prospective study, not a randomized trial. A double blind prospective study with a substantially larger sample size is therefore needed to further validate our findings to evaluate clinical outcome.

\section{Conclusion}

In conclusion, the additional administration of rhTM improved the biomarker levels in patients with survival ARDS, as demonstrated by significant improvements in the $\mathrm{SpO}_{2} / \mathrm{F}_{1} \mathrm{O}_{2}, \mathrm{HMG}-1$ and microvascular coagulation and there was no adverse event. Further clinical investigations such as survival outcome are necessary to evaluate the effect of rhTM on ARDS patients.

\section{REFERENCES}

[1] M. Van de Wouwer, D. Collen and E. M. Conway, "Thrombomodulin-Protein C-EPCR System: Integrated to Regulate Coagulation and Inflammation," Arteriosclerosis, Thrombosis, and Vascular Biology, Vol. 24, 2004, pp. 
1374-1383.

http://dx.doi.org/10.1161/01.ATV.0000134298.25489.92

[2] H. Saito, I. Maruyama, S. Shimazaki, Y. Yamamoto, N. Akikawa, R. Ohno, A. Hirayama, T. Matsuda, H. Asakura, M. Nakashima and N. Aoki, "Efficacy and Safety of Recombinant Human Soluble Thrombomodulin (ART-123) in Disseminated Intravascular Coagulation: Results of a Phase III, Randomized, Double-Blind Clinical Trial," Journal of Thrombosis and Haemostasis, Vol. 5, No. 1, 2007, pp. 31-41.

http://dx.doi.org/10.1111/j.1538-7836.2006.02267.x

[3] S. C. Sebag, J. A. Bastarache and L. B. Ware, "Therapeutic Modulation of Coagulation and Fibrinolysis in Acute Lung Injury and the Acute Respiratory Distress Syndrome," Current Pharmaceutical Biotechnology, Vol. 12, No. 9, 2011, pp. 1481-1496. http://dx.doi.org/10.2174/138920111798281171

[4] J. H. Finigan, "The Coagulation System and Pulmonary Endothelial Function in Actre Lung Injury," Microvascular Research, Vol. 77, No. 1, 2009, pp. 35-38. http://dx.doi.org/10.1016/j.mvr.2008.09.002

[5] H. Lum and A. B. Malik, "Regulation of Vascular Endothelial Barrier Function," American Journal of Physiology -Lung Cellular and Molecular Physiology, Vol. 267, 1994, pp. L233-241.

[6] G. Cirino, C. Cicala, M. R. Bucci, L. Sorrentino, J. M. Maraganore, and S. R. Stone, "Thrombin Functions as an Inflammatory Mediator through Activation of Its Receptor," The Journal of Experimental Medicine, Vol. 183, No. 3, 1996, pp. 821-827. http://dx.doi.org/10.1084/jem.183.3.821

[7] The ARDS Definition Task Force, "Acute Respiratory Distress Syndrome Berlin Definition," JAMA, Vol. 307, No. 23, 2012, pp. 1-8. http://dx.doi.org/10.1001/jama.2012.5669

[8] K. D. Liu, J. Levitt, H. Zhuo, R. H. Kallet, S. Brady, J. Steingrub, M. Tidswell, M. D. Siegel, G. Soto, M. W. Peterson, M. S. Chesnutt, C. Phillips, A. Weinacker, B. T. Thompson, M. D. Eisner and M. A. Matthay, "Randomized Clinical Trial of Activated Protein $\mathrm{C}$ for the Treatment of Acute Lung Injury," American Journal of Respiratory and Critical Care Medicine, Vol. 178, No. 6, 2008, pp. 618-623. http://dx.doi.org/10.1164/rccm.200803-419OC

[9] M. Mohri, Y. Gonda, M. Oka, Y. Aoki, K. Gomi, T. Kiyota, T. Sugihara, S. Yamamoto, T. Ishida and I. Maruyama, "The Antithrombotic Effects of Recombinant Human Soluble Thrombomodulin (rhsTM) on Tissue FactorInduced Disseminated Intravascular Coagulation in Crab-
Eating Monkeys (Macaca fascicularis)," Blood Coagul Fibrinolysis, Vol. 8, 1997, pp. 274-283. http://dx.doi.org/10.1097/00001721-199707000-00003

[10] K. Abeyama, D. M. Stern, Y. Ito, K. Kawahara, Y. Yoshimoto, M. Tanaka, T. Uchimura, N. Ida, Y. Yamazaki, S. Yamada, Y. Yamamoto, H. Yamamoto, S. Iino, N. Taniguchi and I. Maruyama, "The N-Terminal Domain of Thrombomodulin Sequesters High-Mobility Group-B1 Protein, a Novel Antiinflammatory Mechanism," Journal of Clinical Investigation, Vol. 115, No. 5, 2005, pp. 1267 1274.

[11] C. S. Shi, G. Y. Shi, S. M. Hsiao, Y. C. Kao, K. L. Kuo, C. Y. Ma, C. H. Kuo, B. I. Chang, C. F. Chang, C. H. Lin, C. H. Wong and H. L. Wu, "Lectin-Like Domain of Thrombomodulin Binds to Its Specific Ligand Lewis Y Antigen and Neutralizes Lipopolysaccharide-Induced Inflammatory Response,” Blood, Vol. 112, No. 9, 2008, pp. 3661-3670.

[12] M. Van de Wouwer, S. Plaisance, A. De Vriese, E. Waelkens, D. Collen, J. Persson, M. R. Daha and E. M. Conway, "The Lectin-Like Domain of Thrombomodulin Interferes with Complement Activation and Protects against Arthritis," Journal of Thrombosis and Haemostasis, Vol. 4, No. 8, 2006, pp. 1813-1824.

[13] M. Nagato, K. Okamoto, Y. Abe, A. Higure and K. Yamaguchi, "Recombinant Human Soluble Thrombomodulin Decreases the Plasma High-Mobility Group Box-1 Protein Levels, Whereas Improving the Acute Liver Injury and Survival Rates in Experimental Endotoxemia," Critical Care Medicine, Vol. 37, No. 7, 2009, pp. 2181-2186.

[14] E. Abraham, J. Arcaroli, A. Carmody, H. Wang and K. J. Tracey, "HMG-1 as Mediator of Acute Lung Inflammation," The Journal of Immunology, Vol. 165, No. 6, 2000, pp. 2950-2954.

[15] A. Ishizaka, T. Matsuda, K. H. Albertine, H. Koh, S. Tasaka, N. Hasegawa, N. Kohno, T. Kotani, H. Morisaki, J. Takeda, M. Nakamura, X. Fang, T. R. Martin, M. A. Matthay and S. Hashimoto, "Elevation of KL-6, a Lung Epithelial Cell Marker, in Plasma and Epithelial Lining Fluid in Acute Respiratory Distress Syndrome," American Journal of Physiology-Lung Cellular and Molecular Physiology, Vol. 286, 2004, pp. L1088-1094.

[16] P. J. Offner and E. E. Moore, "Lung Injury Severity Scoring in the Era of Lung Protective Mechanical Ventilation: The $\mathrm{PaO}_{2} / \mathrm{F}_{\mathrm{I}} \mathrm{O}_{2}$ Ratio," Journal of Trauma, Vol. 55, No. 2, 2003, pp. 285-289. 